\title{
On Robinsonian dissimilarities, the consecutive ones property and latent variable models
}

\author{
Matthijs J. Warrens
}

Received: 5 August 2008 / Revised: 20 April 2009 / Accepted: 5 June 2009 /

Published online: 24 June 2009

(C) The Author(s) 2009. This article is published with open access at Springerlink.com

\begin{abstract}
A dissimilarity measure on a set of objects is Robinsonian if its matrix can be symmetrically permuted so that its elements do not decrease when moving away from the main diagonal along any row or column. The Robinson property of a dissimilarity reflects an order of the objects. If a dissimilarity is not observed directly, it must be obtained from the data. Given that an ordinal structure is assumed to underlie the data, the dissimilarity function of choice may or may not recover the order correctly. For four dissimilarity measures for binary data it is investigated what ordinal data structure of $0 \mathrm{~s}$ and $1 \mathrm{~s}$ is correctly recovered. We derive sufficient conditions for the dissimilarity functions to be Robinsonian. The sufficient conditions differ with the dissimilarity measures. The paper concludes with some limitations of the study.
\end{abstract}

Keywords Dissimilarity measures - Binary data - Ordinal comparison · Pyramids . Ordered clustering systems · Weakly pseudo-hierarchies

Mathematics Subject Classification (2000) $\quad 62 \mathrm{H} 05 \cdot 62 \mathrm{H} 20$

\section{Introduction}

An important issue in classification and dissimilarity analysis is determining and visualizing relational structures between objects (or individuals). An essential entity in such analysis is a dissimilarity $d$ on a set of objects $E$, which is either observed directly or computed from a data matrix. A dissimilarity $d$ is a function from the Cartesian product $E \times E$ to the nonnegative real numbers such that $d_{i j}=d_{j i}$ and $d_{i i}=0$ for all $i, j \in E$.

\footnotetext{
M. J. Warrens ( $\varangle)$

Unit Methodology and Statistics, Institute of Psychology, Leiden University,

P. O. Box 9555, 2300 RB Leiden, The Netherlands

e-mail: warrens@fsw.leidenuniv.nl
} 
Consider a linear order $\lessdot$ on $E$. We say that $\lessdot$ is compatible with a dissimilarity $d$ on $E$ whenever $i \lessdot j \lessdot k$ implies $d_{i k} \geq \max \left\{d_{i j}, d_{j k}\right\}$ for all $i, j, k \in E$ (Chepoi and Fichet 1997; Barthélemy et al. 2004). A dissimilarity measure $d$ is said to be Robinsonian if it admits a compatible order. Equivalently, $d$ is Robinsonian if its matrix can be symmetrically permuted so that its elements do not decrease when moving away from the main diagonal along any row or column (Critchley and Fichet 1994; Diday 1986). Hubert et al. (1998) use the term anti-Robinsonian for a dissimilarity matrix and reserve the term Robinsonian for a similarity matrix, since Robinson (1951) studied matrices of the similarity type.

Robinsonian dissimilarities play an important role in unidimensional scaling problems in archeology (Robinson 1951; Kendall 1971) and psychology (Hubert 1974; Hubert et al. 1998), in the analysis of DNA sequences (Mirkin and Rodin 1984), and in overlapping clustering (Fichet 1984; Bertrand and Diday 1985; Diday 1986; Mirkin 1996). There is a one-to-one correspondence between Robinsonian dissimilarities and the ordered clustering systems called pyramids in Diday $(1984,1986)$ and pseudohierarchies in Fichet (1984). Critchley and Fichet (1994) discuss some properties and applications of Robinsonian dissimilarities. Some extensions of Robinsonian dissimilarities are discussed in Barthélemy et al. (2004) and Warrens and Heiser (2007).

Given a dissimilarity measure $d$ one may be interested in knowing whether $d$ is Robinsonian. An algorithm for testing whether or not a dissimilarity measure $d$ is Robinsonian is presented in, e.g., Chepoi and Fichet (1997). Recall that $d$ may be either observed directly or may be computed from a data matrix. In the latter case $d$ must be chosen in light of the data analysis, of which it is a part. The choice of $d$ may influence (i) the possibility of a linear order, since certain types of dissimilarity definitions may be more likely to be Robinsonian than others, (ii) the correct recovery of an order, given that an ordinal structure underlies the data. In this paper, we study dissimilarity functions based on binary $(0,1)$ data (Baulieu 1989; Albatineh et al. 2006; Warrens 2008a,b,c,d). Because a large number of dissimilarity functions has been proposed for this type of data in the literature, it is important that the different functions and their properties are better investigated with respect to (i) and (ii).

The paper presents some interesting connections between Robinsonian dissimilarities and several $(0,1)$-data structures. For four dissimilarity measures it is investigated what ordinal data structure of 0 and $1 \mathrm{~s}$ is correctly recovered. The main results are sufficient conditions for a measure to be Robinsonian. The conditions differ with the dissimilarity measures. The results provide some theoretical justification for using a certain dissimilarity measure if a particular data structure can be assumed to underlie the data. A limitation of the study is that the sufficient conditions are rather strong and are often not satisfied with real data (see Sect. 6). In these cases different dissimilarity functions may or may not recover the ordinal structure, and it is difficult to decide what dissimilarity measure to use.

The paper is organized as follows. The next section is used to introduce additional terminology and notation. The four dissimilarity measures that we are studying throughout the paper are presented here. In Sects. 3-5 we consider different types of interesting structures that a $(0,1)$-table may exhibit or that can be assumed to underlie the binary data matrix. Each data structure implies an order on the rows (objects) of the data table. In each section it is checked if a dissimilarity measure is Robinsonian 
and whether or not the linear order is correctly reflected in its dissimilarity matrix. Sect. 6 contains a discussion.

\section{Dissimilarity measures}

Suppose the data are in a binary (0,1)-table $\mathbf{X}=\left\{x_{i l}\right\}$ for $m$ objects (rows) and $n$ attributes (columns), where a value $x_{i l}=1$ denotes that object $i$ exhibits attribute $l$ and a value $x_{i l}=0$ otherwise (see, e.g., Examples 1 and 2). Furthermore, let

$$
p_{i}=\sum_{l=1}^{n} x_{i l}
$$

denote the proportion of attributes that object $i$ exhibits, and let

$$
a_{i j}=\sum_{l=1}^{n} x_{i l} x_{j l}
$$

denote the proportion of attributes that objects $i$ and $j$ have in common. The quantity $p_{i}$ is the proportion of $1 \mathrm{~s}$ in the $i$ th row of $\mathbf{X}$, whereas quantity $a_{i j}$ is the proportion of $1 \mathrm{~s}$ that rows $i$ and $j$ share in the same positions. We have $p_{i}, p_{j} \geq a_{i j}$ and $a_{i i}=p_{i}$.

Resemblance measures for two binary $(0,1)$-sequences $i$ and $j$ are discussed in Gower and Legendre (1986, Section 4.1), Baulieu (1989), Batagelj and Bren (1995, Sect. 4), Albatineh et al. (2006) and Warrens (2008a,b,c,d). We consider just four functions from the vast amount of measures that has been proposed in the literature. In the present notation, the complement of the simple matching coefficient (Sokal and Michener 1958), also known as the misclassification rate, can be written as

$$
d_{i j}^{\mathrm{SM}}=p_{i}+p_{j}-2 a_{i j}
$$

Dissimilarity measures

$$
\begin{aligned}
d_{i j}^{\mathrm{RR}} & = \begin{cases}1-a_{i j} & \text { for } i \neq j \\
0 & \text { for } i=j\end{cases} \\
d_{i j}^{\mathrm{B}} & =1-\frac{a_{i j}}{\max \left(p_{i}, p_{j}\right)}, \quad \text { and } \\
d_{i j}^{\mathrm{J}} & =1-\frac{a_{i j}}{p_{i}+p_{j}-a_{i j}}
\end{aligned}
$$

are the complements of the Russel and Rao (1940), Braun-Blanquet (1932) and Jaccard (1912) similarity coefficients, respectively. Let $\mathbf{D}^{\mathrm{RR}}$ denote the dissimilarity matrix corresponding to $d^{\mathrm{RR}}$.

Although there are many other possible functions for binary data, there are several reasons to limit this study to the above four dissimilarity measures. Functions $d^{\mathrm{SM}}$ 
and $d^{\mathrm{J}}$ are popular dissimilarity measures that have been studied and applied extensively in various domains of data analysis. Moreover, both measures are a prototype member of one of the two parameter families studied in Gower (1986) and Gower and Legendre (1986). For example, function $d^{\mathrm{J}}$ belongs to the same parameter family as the well-known Dice (1945) coefficient $2 a_{i j} /\left(p_{i}+p_{j}\right)$. Two members of any of these parameter families are globally order equivalent (Sibson 1972), i.e., they are interchangeable with respect to an analysis method that is invariant under ordinal transformations. This means that $d^{\mathrm{J}}$ is Robinsonian if and only if the matrix with elements $1-\left[2 a_{i j} /\left(p_{i}+p_{j}\right)\right]($ size $m \times m)$ is Robinsonian. Finally, for functions $d^{\mathrm{RR}}$ (Sect. 3) and $d^{\mathrm{B}}$ (Sect.4) we consider a sufficient condition that appears to be unique to these coefficients.

\section{Consecutive 1s property}

Recall that the data are in a binary $(0,1)$-table $\mathbf{X}=\left\{x_{i l}\right\}$ of size $m \times n$. A $(0,1)$-table has the consecutive ones property $(\mathrm{C} 1 \mathrm{P})$ for columns when there is a permutation of its rows that arranges the $1 \mathrm{~s}$ at consecutive positions in every column, i.e., in each column all 1s form a contiguous sequence (Meidanis et al. 1998). One can analogously define the $\mathrm{C} 1 \mathrm{P}$ for rows. The $\mathrm{C} 1 \mathrm{P}$ appears naturally in a wide range of applications (Booth and Lueker 1976; Ghosh 1972; Greenberg and Istrail 1995). There is an extensive literature on the graph-theoretical characterization of tables with consecutive $1 \mathrm{~s}$ (Meidanis et al. 1998; Kendall 1969; Hubert 1974) and algorithms to identify it (Booth and Lueker 1976; Hsu 2002). The C1P can be used to recognize interval hypergraphs (Fulkerson and Gross 1965).

We consider the following result by Kendall (1969).

Lemma 1 [Kendall 1969] Suppose the 1s are consecutive in every column of the data table. Then $i<j<k$ implies $a_{i j} \geq a_{i k}$ and $a_{i k} \leq a_{j k}$.

Proof If the columns contain consecutive 1s, then the objects (rows) $i, j$ and $k$ can form the six types of column profiles

\begin{tabular}{lcccccc}
\hline$i$ & 1 & 0 & 0 & 1 & 0 & 1 \\
$j$ & 0 & 1 & 0 & 1 & 1 & 1 \\
$k$ & 0 & 0 & 1 & 0 & 1 & 1 \\
\hline freq. & $u_{1}$ & $u_{2}$ & $u_{3}$ & $u_{4}$ & $u_{5}$ & $u_{6}$
\end{tabular}

with (absolute) frequencies $u_{1}-u_{6}$. Thus, $u_{1}$ is the number of column profiles that contain a 1 for object $i$ and a 0 for objects $j$ and $k$. We have $a_{i j} \geq a_{i k}$ if and only if $u_{4}+u_{6} \geq u_{6}$. Since $u_{4}$ and $u_{6}$ are frequencies, inequality $u_{4}+u_{6} \geq u_{6}$ always holds. Furthermore, we have $a_{i k} \leq a_{j k}$ if and only if $u_{6} \leq u_{5}+u_{6}$. This completes the proof.

Theorem 1 is a direct consequence of Lemma 1. The result is due to Kendall (1969) and connects Robinsonian dissimilarities to interval hypergraphs. 
Theorem 1 [Kendall 1969] If the data matrix has the C1P for the columns, then $d^{R R}$ is Robinsonian.

Proof We have $d_{i k}^{\mathrm{RR}} \geq d_{i j}^{\mathrm{RR}}$ if and only if $a_{i k} \leq a_{i j}$ and $d_{i k}^{\mathrm{RR}} \geq d_{j k}^{\mathrm{RR}}$ if and only if $a_{i k} \leq a_{j k}$. The assertion then follows from application of Lemma 1 .

Example 1 The property in Theorem 1 appears to be unique to $d^{\mathrm{RR}}$. Consider the following data table with four objects and seven attributes:

\begin{tabular}{lllllll}
\hline 1 & 0 & 0 & 0 & 0 & 0 & 0 \\
1 & 1 & 1 & 0 & 0 & 0 & 0 \\
0 & 0 & 1 & 1 & 1 & 1 & 0 \\
0 & 0 & 1 & 0 & 0 & 1 & 1 \\
\hline
\end{tabular}

This table has the C1P for the columns. The dissimilarity matrices of the four dissimilarity measures from Sect. 2 are

\begin{tabular}{cccc}
\hline 0 & 0.86 & 1.00 & 1.00 \\
& 0 & 0.86 & 0.86 \\
& & 0 & 0.71 \\
$\mathbf{D}^{\mathrm{RR}}$ & & & 0 \\
\hline
\end{tabular}

\begin{tabular}{cccc}
\hline 0 & 0.29 & 0.71 & 0.57 \\
& 0 & 0.71 & 0.57 \\
& & 0 & 0.43
\end{tabular}

$\mathbf{D}^{\mathrm{SM}}$

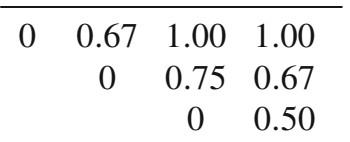

$\underline{\mathbf{D}^{\mathrm{B}}}$

\begin{tabular}{cccc}
\hline 0 & 0.67 & 1.00 & 1.00 \\
& 0 & 0.83 & 0.80 \\
& & 0 & 0.60 \\
$\mathbf{D}^{\mathrm{J}}$ & & & 0 \\
\hline
\end{tabular}

Measure $d^{\mathrm{RR}}$ is Robinsonian. The given order of the objects is compatible with $d^{\mathrm{RR}}$. The matrices of $d^{\mathrm{B}}, d^{\mathrm{SM}}$ and $d^{\mathrm{J}}$ are not Robinsonian.

Example 1 shows that measures $d^{\mathrm{J}}$ and $d^{\mathrm{B}}$ are not necessarily Robinsonian when the data table has the C1P for the columns. The two dissimilarity measures $d^{\mathrm{J}}$ and $d^{\mathrm{B}}$ are Robinsonian under stronger conditions (Theorems 2 and 3). We first present the following lemma.

Lemma 2 Suppose the $1 s$ are consecutive in every column of the data table. Furthermore, suppose that the data table has the C1P for the rows. Then $i<j<k$ implies

$$
\frac{a_{i j}}{p_{j}} \geq \frac{a_{i k}}{p_{k}} \text { and } \frac{a_{i k}}{p_{i}} \leq \frac{a_{j k}}{p_{j}}
$$

Proof We can distinguish two situations under the conditions of the assertion, one that contains the column profile $\left(\begin{array}{lll}1 & 1 & 1\end{array}\right)^{\prime}$, and one with $\left(\begin{array}{lll}0 & 1 & 0\end{array}\right)^{\prime}$. In the first situation rows 
$i, j$ and $k$ form the five types of column profiles

\begin{tabular}{lccccc}
\hline$i$ & 1 & 0 & 1 & 0 & 1 \\
$j$ & 0 & 0 & 1 & 1 & 1 \\
$k$ & 0 & 1 & 0 & 1 & 1 \\
\hline freq. & $u_{1}$ & $u_{2}$ & $u_{3}$ & $u_{4}$ & $u_{5}$
\end{tabular}

with frequencies $u_{1}-u_{5}$. We have

$$
\begin{aligned}
\frac{a_{i j}}{p_{j}} & \geq \frac{a_{i k}}{p_{k}} \\
\frac{u_{3}+u_{5}}{u_{3}+u_{4}+u_{5}} & \geq \frac{u_{5}}{u_{2}+u_{4}+u_{5}} \\
u_{2} u_{3}+u_{3} u_{4}+u_{2} u_{5} & \geq 0 .
\end{aligned}
$$

In the second situation the objects $i, j$ and $k$ form the five types of column profiles

\begin{tabular}{lccccc}
\hline$i$ & 1 & 0 & 0 & 1 & 0 \\
$j$ & 0 & 1 & 0 & 1 & 1 \\
$k$ & 0 & 0 & 1 & 0 & 1 \\
\hline freq. & $v_{1}$ & $v_{2}$ & $v_{3}$ & $v_{4}$ & $v_{5}$
\end{tabular}

with frequencies $v_{1}-v_{5}$. We have

$$
\begin{aligned}
\frac{a_{i j}}{p_{j}} & \geq \frac{a_{i k}}{p_{k}} \\
\frac{v_{4}}{v_{2}+v_{4}+v_{5}} & \geq \frac{0}{v_{3}+v_{5}} .
\end{aligned}
$$

This completes the proof for the first inequality. The second inequality follows from using similar arguments. This completes the proof.

Theorem 2 If the data table has the C1P for both the rows and columns, then $d^{J}$ is Robinsonian.

Proof Under the conditions of the theorem, $i<j<k$ implies $d_{i k}^{\mathrm{J}} \geq d_{i j}^{\mathrm{J}}$ and $d_{i k}^{\mathrm{J}} \geq$ $d_{j k}^{\mathrm{J}}$. In fact, by Lemmas 1 and 2 we have $p_{i} a_{i k} \leq p_{i} a_{i j}$ and $p_{j} a_{i k} \leq p_{k} a_{i j}$. Adding the two inequalities we obtain

$$
\begin{aligned}
\frac{a_{i k}}{p_{i}+p_{k}} & \leq \frac{a_{i j}}{p_{i}+p_{j}} \\
\frac{a_{i k}}{p_{i}+p_{k}-a_{i k}} & \leq \frac{a_{i j}}{p_{i}+p_{j}-a_{i j}} \\
d_{i k}^{\mathrm{J}} & \geq d_{i j}^{\mathrm{J}} .
\end{aligned}
$$

Inequality $d_{i k}^{\mathrm{J}} \geq d_{j k}^{\mathrm{J}}$ follows from using similar arguments. This completes the proof. 
Theorem 3 If the data table has the C1P for both the rows and columns, then $d^{B}$ is Robinsonian.

Proof Under the conditions of the theorem, $i<j<k$ implies $d_{i k}^{\mathrm{B}} \geq d_{i j}^{\mathrm{B}}$ and $d_{i k}^{\mathrm{B}} \geq d_{j k}^{\mathrm{B}}$. We have $d_{i k}^{\mathrm{B}} \geq d_{i j}^{\mathrm{B}}$ if and only if

$$
\frac{a_{i j}}{\max \left(p_{i}, p_{j}\right)} \geq \frac{a_{i k}}{\max \left(p_{i}, p_{k}\right)} .
$$

Probabilities $p_{i}, p_{j}$ and $p_{k}$ can be ordered in six different ways. If $p_{i} \geq p_{j}, p_{k}$, then (3) follows from Lemma 1. If $p_{i} \leq p_{j}, p_{k}$, then (3) follows from Lemma2. There are two more cases to examine.

If $p_{j} \geq p_{i} \geq p_{k}$, then (3) becomes

$$
\frac{a_{i j}}{p_{j}} \geq \frac{a_{i k}}{p_{i}} .
$$

Inequality (4) must be checked for the two situations in the proof of Lemma2. The second situation is straightforward (since $a_{i k}=0$ ). For the first situation we have

$$
\begin{aligned}
\frac{a_{i j}}{p_{j}} & \geq \frac{a_{i k}}{p_{i}} \\
\frac{u_{3}+u_{5}}{u_{3}+u_{4}+u_{5}} & \geq \frac{u_{5}}{u_{1}+u_{3}+u_{5}} \\
\left(u_{1}+u_{3}\right)\left(u_{3}+u_{5}\right) & \geq u_{4} u_{5} .
\end{aligned}
$$

Since $p_{i} \geq p_{k}$, we have

$$
\begin{aligned}
u_{1}+u_{3} & \geq u_{2}+u_{4} \\
\left(u_{1}+u_{3}\right) u_{5} & \geq\left(u_{2}+u_{4}\right) u_{5} \geq u_{4} u_{5},
\end{aligned}
$$

which implies inequality (5).

If $p_{k} \geq p_{i} \geq p_{j}$, then (3) becomes

$$
\frac{a_{i j}}{p_{i}} \geq \frac{a_{i k}}{p_{k}} .
$$

Inequality (6) follows from Lemma $1\left(a_{i j} \geq a_{i k}\right)$ and $p_{k} \geq p_{i}$. This completes the proof of $d_{i k}^{\mathrm{B}} \geq d_{i j}^{\mathrm{B}}$. Inequality $d_{i k}^{\mathrm{B}} \geq d_{j k}^{\mathrm{B}}$ follows from using similar arguments.

Example 2 Consider the following data table with four objects and five attributes:

\begin{tabular}{lllll}
\hline 1 & 1 & 0 & 0 & 0 \\
0 & 1 & 1 & 1 & 0 \\
0 & 0 & 1 & 1 & 1 \\
0 & 0 & 0 & 0 & 1 \\
\hline
\end{tabular}


This table has the C1P for both the rows and columns. The dissimilarity matrices corresponding to $d^{\mathrm{SM}}$ and $d^{\mathrm{J}}$ are

\begin{tabular}{cccc}
\hline 0 & 0.60 & 1.00 & 0.60 \\
& 0 & 0.40 & 0.80 \\
& & 0 & 0.40 \\
$\mathbf{D}^{\mathrm{SM}}$ & & & 0
\end{tabular}

\begin{tabular}{cccc}
\hline 0 & 0.75 & 1.00 & 1.00 \\
& 0 & 0.50 & 1.00 \\
& & 0 & 0.67 \\
$\mathbf{D}^{\mathrm{J}}$ & & & 0 \\
\hline
\end{tabular}

Function $d^{\mathrm{J}}$ is Robinsonian. The given order of the objects is compatible with $d^{\mathrm{J}}$. Dissimilarity measure $d^{\mathrm{SM}}$ is not Robinsonian. Measure $d^{\mathrm{SM}}$ is thus not necessarily Robinsonian when the data table has the C1P for both the rows and columns.

\section{Monotone functions}

In Sects. 4 and 5 we assume that a latent variable model underlies the $(0,1)$-data table $\mathbf{X}$. The elements $x_{i l}=0,1$ are now realizations under a latent variable model. Let $\theta$ be a latent variable. In Sects. 4 and 5 we assume that for each object (row) $i$ the value 1 is modeled by a probabilistic function of $\theta$, denoted by $p_{i}(\theta)$, with $0 \leq p_{i}(\theta) \leq 1$. In item response theory (Van der Linden and Hambleton 1997; Sijtsma and Molenaar 2002), $p_{i}(\theta)$ is called the item response function or the item characteristic curve of item $i$. The value 0 in row $i$ is modeled by the function $1-p_{i}(\theta)$. The four dissimilarity functions in Sect. 2 are defined in terms of $p_{i}$ and $a_{i j}$. In Sects. 4 and 5, the definitions of $p_{i}$ and $a_{i j}$ in (7) and (8) replace the definitions in (1) and (2). Let us show how quantities $p_{i}$ and $a_{i j}$ are related to the $p_{i}(\theta)$.

Let $L(\theta)$ denote the probability density function of the latent variable $\theta$. Function $L(\theta)$ specifies how the attributes are distributed over the latent variable $\theta$. Here, we do not require that $L(\theta)$ has a particular form, and the results in this section hold for any choice of $L(\theta)$.

The unconditional probability of a value 1 for object (row) $i$ is given by

$$
p_{i}=\int_{\mathbb{R}} p_{i}(\theta) d L(\theta),
$$

where $\mathbb{R}$ denotes the set of reals. Next, we assume that conditionally on $\theta$ the presence or absence of an attribute for different rows (objects) of the data matrix $\mathbf{X}$ are stochastically independent. The joint probability of 1 s of objects $i$ and $j$, given a value of $\theta$, is then given by $p_{i}(\theta) p_{j}(\theta)$. The corresponding unconditional probability can be obtained from

$$
a_{i j}=\int_{\mathbb{R}} p_{i}(\theta) p_{j}(\theta) d L(\theta) .
$$

If we would like to estimate $p_{i}$ and $a_{i j}$ for real data, the quantities in (1) and (2) could be used as estimates. 
In this section, we suppose that the functions $p_{i}(\theta)$ are monotonically increasing on the continuum $\theta$, i.e.,

$$
p_{i}\left(\theta_{1}\right) \leq p_{i}\left(\theta_{2}\right) \text { for } \theta_{1}<\theta_{2} \text {. }
$$

If (9) holds, then 1s are more probable for high values than for low values of $\theta$.

In addition to (9), suppose that the objects (rows of the data table) can be ordered such that the corresponding functions $p_{i}(\theta)$ are non intersecting on the whole range of the continuum $\theta$, i.e.,

$$
p_{i}(\theta) \geq p_{j}(\theta) \text { for } i<j
$$

If (10) holds, then $1 \mathrm{~s}$ are more probable in row $i$ than in row $j$.

The case that assumes (9) and (10), together with the assumptions of local independence and a single latent variable, is called the double monotonicity model in nonparametric item response theory (Sijtsma and Molenaar 2002). A well-known result is that, if the double monotonicity model holds, then the objects (rows of $\mathbf{X}$ ) can be ordered such that we have

$$
p_{i} \geq p_{j} \text { for } i<j
$$

and

$$
a_{i k} \geq a_{j k} \text { for } i<j, k \neq j .
$$

If (11) holds, then row $i$ contains more $1 \mathrm{~s}$ than row $j$. If (12) holds, then rows $i$ and $k$ share more $1 \mathrm{~s}$ in the same positions (columns) than rows $j$ and $k$.

Apart from being monotonically increasing, functions $p_{i}(\theta)$ may also satisfy various orders of total positivity (Karlin 1968). Total positivity is a very general concept, but it can also be formulated for a set of functions $p_{i}(\theta)$ (Schriever 1986; Post 1992). If a set of functions $p_{i}(\theta)$ is totally positive of order 2 , then the objects can be ordered such that

$$
p_{i}\left(\theta_{1}\right) p_{j}\left(\theta_{2}\right)-p_{i}\left(\theta_{2}\right) p_{j}\left(\theta_{1}\right) \geq 0 \text { for } \theta_{1}<\theta_{2} \text { and } i<j \text {. }
$$

Example 3 The response function of the one-parameter logistic or Rasch (1960) model is given by

$$
p_{i}^{\mathrm{R}}\left(\theta, b_{i}\right)=\frac{\exp \left(\theta-b_{i}\right)}{1+\exp \left(\theta-b_{i}\right)},
$$

where 'exp' is the exponential function and $b_{i}$ is the location parameter. In item response theory (Van der Linden and Hambleton 1997) parameter $b_{i}$ is also called the difficulty parameter. A set of functions $p_{i}^{\mathrm{R}}\left(\theta, b_{i}\right)$ is called a location family, since the $p_{i}^{\mathrm{R}}\left(\theta, b_{i}\right)$ have the same shape and only differ in their location $\left(b_{i}\right)$ on the latent 
variable $\theta$. The location family of functions $p_{i}^{\mathrm{R}}\left(\theta, b_{i}\right)$ satisfies conditions (9), (10) and (13).

Schriever (1986) derived the following result for a set of functions that are both monotonically increasing and satisfy total positivity of order 2 . The proof is presented for completeness.

Lemma 3 [Schriever 1986] If the objects are ordered such that (9) and (13) hold, then

$$
\frac{a_{i k}}{p_{i}} \leq \frac{a_{j k}}{p_{j}} \text { for } i<j, k \neq i
$$

Proof $p_{i}^{-1} p_{i}(\theta)$ can be interpreted as a density with respect to the measure $L$, which by (13), is totally positive of order 2 and satisfies

$$
\int_{\mathbb{R}} \frac{p_{j}(\theta)}{p_{j}} d L(\theta)=1 .
$$

Since by (9), $p_{j}(\theta)$ is increasing in $\theta$ for each $j$, it follows from Proposition 3.1 in Karlin (1968, p. 22) that

$$
\frac{a_{i j}}{p_{i}}=\int_{\mathbb{R}} \frac{p_{i}(\theta) p_{j}(\theta)}{p_{i}} d L(\theta)
$$

is increasing in $i$.

Dissimilarity measure $d^{\mathrm{B}}$ is Robinsonian if the rows of the data table can be permuted such that (9), (10) and (13) hold.

Theorem 4 Suppose the rows of the data table can be permuted such that (9), (10) and (13) hold. Then $d^{B}$ is Robinsonian.

Proof It must be shown that, under the conditions of the theorem, $i<j<k$ implies $d_{i k}^{\mathrm{B}} \geq \max \left\{d_{i j}^{\mathrm{B}}, d_{j k}^{\mathrm{B}}\right\}$. First note that under these conditions (11), (12) and (14) hold. Due to (11), we have

$$
\begin{aligned}
d_{i k}^{\mathrm{B}} & \geq d_{i j}^{\mathrm{B}} \\
\frac{a_{i k}}{\max \left(p_{i}, p_{k}\right)} & \leq \frac{a_{i j}}{\max \left(p_{i}, p_{j}\right)} \\
\frac{a_{i k}}{p_{i}} & \leq \frac{a_{i j}}{p_{i}} .
\end{aligned}
$$

Inequality (15) follows from (12). 
Next it must be shown that $d_{i k}^{\mathrm{B}} \geq d_{j k}^{\mathrm{B}}$. Due to (11), we have

$$
\begin{aligned}
d_{i k}^{\mathrm{B}} & \geq d_{j k}^{\mathrm{B}} \\
\frac{a_{i k}}{\max \left(p_{i}, p_{k}\right)} & \leq \frac{a_{j k}}{\max \left(p_{j}, p_{k}\right)} \\
\frac{a_{i k}}{p_{i}} & \leq \frac{a_{j k}}{p_{j}}
\end{aligned}
$$

which is equivalent to (14). This completes the proof.

Example 4 Five binary sequences were generated using the Rasch function (Example 3) with location parameters $b_{i}=\{-2,-1,0,1,2\}$ and $L(\theta) \sim N(0,1)$ (standard normal distribution). The objects were ordered on the location parameters. The four dissimilarity matrices are

\begin{tabular}{ccccc}
\hline 0 & 0.37 & 0.57 & 0.71 & 0.86 \\
& 0 & 0.62 & 0.75 & 0.86 \\
& & 0 & 0.81 & 0.90 \\
& & & 0 & 0.92 \\
$\mathbf{D}^{\mathrm{RR}}$ & & & & 0 \\
\hline & & & & \\
& & & & \\
\hline 0 & 0.31 & 0.48 & 0.59 & 0.72 \\
& 0 & 0.44 & 0.53 & 0.60 \\
& & 0 & 0.42 & 0.44 \\
& & & 0 & 0.30 \\
$\mathbf{D}^{\mathrm{SM}}$ & & & & 0 \\
\hline
\end{tabular}

\begin{tabular}{ccccc}
\hline 0 & 0.26 & 0.50 & 0.66 & 0.83 \\
& 0 & 0.47 & 0.65 & 0.81 \\
& & 0 & 0.61 & 0.79 \\
& & & 0 & 0.75
\end{tabular}

$\underline{\mathbf{D}^{\mathrm{B}}}$

\begin{tabular}{ccccc}
\hline 0 & 0.33 & 0.52 & 0.67 & 0.84 \\
& 0 & 0.53 & 0.68 & 0.81 \\
& & 0 & 0.69 & 0.81 \\
& & & 0 & 0.80
\end{tabular}

$\underline{\mathbf{D}^{\mathrm{J}}} \quad 0$

Both $d^{\mathrm{B}}$ and $d^{\mathrm{SM}}$ are Robinsonian and the order of the objects (location parameters) is compatible with $d^{\mathrm{B}}$ and $d^{\mathrm{SM}}$. Functions $d^{\mathrm{RR}}$ and $d^{\mathrm{J}}$ are not necessarily Robinsonian when the data is generated using the Rasch model.

Remark Note that $d^{\mathrm{SM}}$ is Robinsonian for the generated data in Example 4. Let us show when this property fails. Let $u_{i j k}^{110}$ denote the number of attributes (frequency) that objects $i$ and $j$ possess and object $k$ lacks. For $i<j<k$, we have

$$
\begin{aligned}
d_{i k}^{\mathrm{SM}} & \geq d_{i j}^{\mathrm{SM}} \\
p_{k}-2 a_{i k} & \geq p_{j}-2 a_{i j} .
\end{aligned}
$$

Using $n \times p_{k}=u_{i j k}^{001}+u_{i j k}^{101}+u_{i j k}^{011}+u_{i j k}^{111}$ and $n \times a_{i k}=u_{i j k}^{101}+u_{i j k}^{111}$, where $n$ is the total number of attributes, (16) becomes

$$
u_{i j k}^{001}+u_{i j k}^{110} \geq u_{i j k}^{010}+u_{i j k}^{101} .
$$


Of the four frequencies in (17), $u_{i j k}^{110}$ represents the only so-called Guttman profile $\left(\begin{array}{lll}1 & 1 & 0\end{array}\right)^{\prime}$ in (17). For these data the profile $\left(\begin{array}{lll}1 & 1 & 0\end{array}\right)^{\prime}$ is the most abundant. Dissimilarity measure $d^{\mathrm{SM}}$ does not reflect the correct order if inequality (17) is false. Nevertheless, it appears that $d^{\mathrm{SM}}$ is more likely to be Robinsonian with monotone latent variable models than either $d^{\mathrm{RR}}$ and $d^{\mathrm{J}}$.

\section{Unimodal functions}

Apart from being monotonically increasing, functions $p_{i}(\theta)$ may also have a unimodal or single-peaked shape (Andrich 1988; Hoijtink 1990; Andrich and Luo 1993; Post and Snijders 1993). A function $p_{i}(\theta)$ is unimodal if for some value $\theta_{0}$ (the mode), it is monotonically increasing for $\theta \leq \theta_{0}$ and monotonically decreasing for $\theta_{0} \geq \theta$. The maximum value of $p_{i}(\theta)$ is $p_{i}\left(\theta_{0}\right)$ and there are no other local maxima. The value $\theta_{0}$ may be considered the location of object $i$ on the latent variable $\theta$ if the function $p_{i}(\theta)$ is symmetric.

A set of functions may form a location family. These functions have a common shape (and maximum) and only differ in their location on the latent variable $\theta$. Many probability density functions may be used to create a location family. We consider two examples that come from unimodal item response theory (Post 1992; Andrich and Luo 1993).

Example 5 The response function that characterizes the squared simple logistic model (Andrich 1988; Post 1992) is defined as

$$
p_{i}^{\mathrm{A}}\left(\theta, b_{i}\right)=\frac{\exp \left[-\left(\theta-b_{i}\right)^{2}\right]}{1+\exp \left[-\left(\theta-b_{i}\right)^{2}\right]},
$$

where $b_{i}$ is the location parameter. The unimodal function is bell-shaped and has a maximum value of 0.5 , assumed for $\theta=b_{i}$. The symmetric functions $p_{i}^{\mathrm{A}}\left(\theta, b_{i}\right)$ have the same shape and only differ in their location $\left(b_{i}\right)$ on the latent variable $\theta$. A set of functions $p_{i}^{\mathrm{A}}\left(\theta, b_{i}\right)$ can thus be considered a location family.

Example 6 Function

$$
p_{i}^{\mathrm{C}}\left(\theta, b_{i}\right)=\frac{1}{1+\left(\theta-b_{i}\right)^{2}}
$$

is the Cauchy function, where $b_{i}$ is the location parameter. Function $p_{i}^{\mathrm{C}}\left(\theta, b_{i}\right)$ is the basic building block of the model proposed in Hoijtink $(1990,1991)$. The unimodal and symmetric function $p_{i}^{\mathrm{C}}\left(\theta, b_{i}\right)$ has a maximum value of 1 .

In the previous section we reviewed essential requirements for monotone functions (Eqs. (9), (10) and (13)) without making specific assumptions concerning the form of the response functions. Post (1992) and Post and Snijders (1993) have formulated such requirements for unimodal functions, using the concept of total positivity (Karlin 1968). We have found no sufficient conditions for a dissimilarity function to 
be Robinsonian when unimodal functions can be assumed to underlie the data. The following example shows that measure $d^{\mathrm{J}}$ reflects the correct ordering of a location family with unimodal functions.

Example 7 Five binary sequences were generated using the Cauchy function (Example 6) with location parameters $b_{i}=\{-2,-1,0,1,2\}$ and $L(\theta) \sim N(0,0.5)$. The objects were ordered on the location parameters. The four dissimilarity matrices are

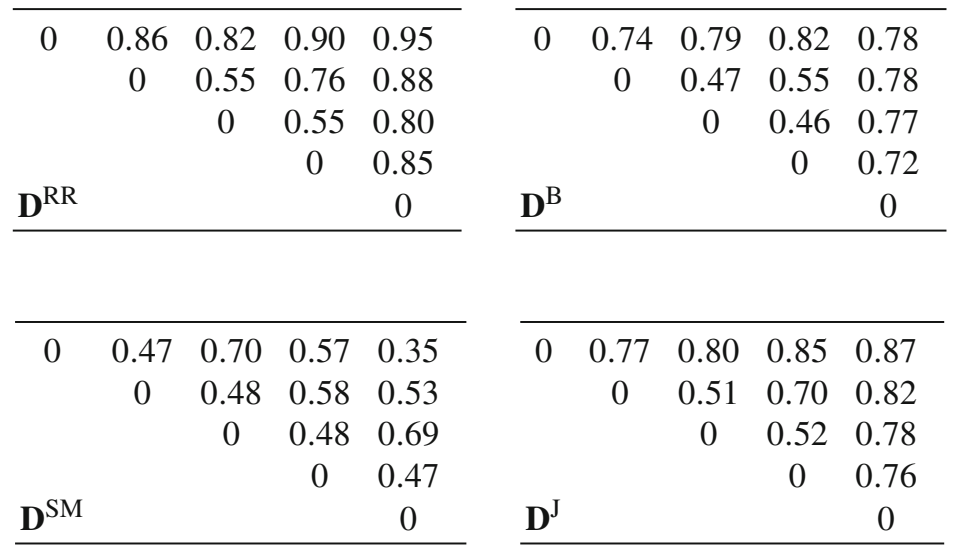

Dissimilarity measure $d^{\mathrm{J}}$ is Robinsonian and the order of the objects (location parameters) is compatible with $d^{\mathrm{J}}$. The other matrices do not reflect the order of the location parameters. In unreported simulation studies it was found that $d^{\mathrm{J}}$ is Robinsonian for various different location families and various probability density functions (normal, uniform, skewed, bimodal) of the latent variable $\theta$. Moreover, the other three dissimilarity measures consistently fail to reflect the correct order of the objects.

\section{Discussion}

A dissimilarity on a set of objects is Robinsonian if its matrix can be symmetrically permuted so that its elements do not decrease when moving away from the main diagonal along any row or column. The Robinson property of a dissimilarity reflects an order of the objects, but also constitutes a clustering system with overlapping clusters. In this paper, we presented some connections between Robinsonian dissimilarities and several $(0,1)$-data structures. For four dissimilarity measures it was investigated what ordinal data structure of 0 s and $1 \mathrm{~s}$ is correctly recovered. The main results are sufficient conditions for the dissimilarity measures to be Robinsonian. The conditions differ with the measures. The results provide a theoretical basis for using certain dissimilarity functions if a particular data structure can be assumed to underlie the data.

Two types of ordinal data structures for $(0,1)$-data were considered: consecutive ones and latent variable models. A $(0,1)$-table has the consecutive ones property for 
columns when there is a permutation of its rows that leaves the $1 \mathrm{~s}$ consecutive in every column, i.e., the $1 \mathrm{~s}$ in a column form a consecutive interval. The consecutive ones property appears naturally in a wide range of applications (Booth and Lueker 1976; Ghosh 1972; Greenberg and Istrail 1995). There is an extensive literature on the graph-theoretical characterization of tables with consecutive 1s (Meidanis et al. 1998; Kendall 1969; Hubert 1974) and algorithms to identify it (Booth and Lueker 1976; Hsu 2002).

Latent variable models are employed in a variety of fields of science, including biological ecology and psychometrics, but are particularly used in item response theory (Van der Linden and Hambleton 1997; Sijtsma and Molenaar 2002). Models with monotone functions (Example 3) are often used for measuring ability, whereas models with unimodal functions (Examples 5 and 6) are more suitable for measuring attitude. The consecutive ones property and latent variable models are conceptually two different things. The former can be observed (after appropriate permutations), whereas the latter are assumed to underlie the data. If the $(0,1)$-table has the consecutive ones property for the objects, the consecutive ones can be interpreted in terms of a deterministic latent variable model (Lazarsfeld and Henry 1968; Coombs 1964).

A limitation of the study is that the sufficient conditions are rather strong and are often not satisfied with real data. In these cases different dissimilarity functions may or may not recover the ordinal structure, and it is at present unclear what dissimilarity should be preferred. For example, Example 7 showed that dissimilarity measure $d^{\mathrm{J}}$ may be used to recover the correct order (e.g., in terms of the maximums or peaks) for location families with unimodal functions. This does not mean that $d^{\mathrm{J}}$ always recovers the correct order if it assumed that unimodal response functions are most appropriate for the data at hand. Moreover, in Sect. 4 it was shown (Theorem 4 and Example 4 ) that measure $d^{\mathrm{B}}$ is perhaps best suitable for location families with monotone functions. However, this does not mean that $d^{\mathrm{B}}$ cannot be useful when applied to a data structure based on unimodal functions. These considerations are illustrated with real data in the following example.

Example 8 The data in Formann (1988, p. 56) are the responses of 600 persons on five dichotomous items concerning the attitude toward nuclear power. The responses were 'I agree' and 'I do not agree'. The items are

1. In the near future, alternate sources of energy will not be able to substitute nuclear energy.

2. It is difficult to decide between the different types of power stations if one carefully considers all their pros and cons.

3. Nuclear power stations should not be put into operation before the problems of radioactive waste have been solved.

4. Nuclear power stations should not be put into operation before it is proven that the radiation caused by them is harmless.

5. The foreign power stations now in operation should be closed down.

The content of the items suggests that a model with unimodal functions is appropriate for these data. Furthermore, the ordering of the items corresponds to the ordering that is reflected in the contents of the items: positive responses to item 1 indicate the most 
favorable attitude toward nuclear energy, and positive responses to item 5 the most disapproving attitude (Formann 1988). The four dissimilarity matrices are

\begin{tabular}{ccccc}
\hline 0 & 0.83 & 0.71 & 0.77 & 0.92 \\
& 0 & 0.59 & 0.62 & 0.82 \\
& & 0 & 0.31 & 0.63 \\
& & & 0 & 0.53 \\
$\mathbf{D}^{\mathrm{RR}}$ & & & & 0 \\
\hline & & & & \\
& & & & \\
\hline 0 & 0.56 & 0.43 & 0.33 & 0.35 \\
& 0 & 0.53 & 0.48 & 0.40 \\
& & 0 & 0.74 & 0.42 \\
& & & 0 & 0.62 \\
$\mathbf{D}^{\mathrm{SM}}$ & & & & 0 \\
\hline
\end{tabular}

\begin{tabular}{ccccc}
\hline 0 & 0.63 & 0.65 & 0.71 & 0.83 \\
& 0 & 0.51 & 0.54 & 0.64 \\
& & 0 & 0.17 & 0.55 \\
& & & 0 & 0.42 \\
$\mathbf{D}^{\mathrm{B}}$ & & & & 0 \\
\hline
\end{tabular}

\begin{tabular}{ccccc}
\hline 0 & 0.72 & 0.67 & 0.74 & 0.89 \\
& 0 & 0.54 & 0.58 & 0.77 \\
& & 0 & 0.27 & 0.61 \\
& & & 0 & 0.45 \\
$\mathbf{D}^{\mathrm{J}}$ & & & & 0 \\
\hline
\end{tabular}

It can be seen that dissimilarity measure $d^{\mathrm{B}}$ is Robinsonian, and the order of the items as suggested in Formann (1988), is compatible with $d^{\mathrm{B}}$. Function $d^{\mathrm{J}}$, despite Example 7, and $d^{\mathrm{SM}}$ and $d^{\mathrm{RR}}$ are not Robinsonian.

Acknowledgments The author would like to thank Hans-Hermann Bock, Maurizio Vichi and two anonymous reviewers for their helpful comments and valuable suggestions on earlier versions of this article.

Open Access This article is distributed under the terms of the Creative Commons Attribution Noncommercial License which permits any noncommercial use, distribution, and reproduction in any medium, provided the original author(s) and source are credited.

\section{References}

Albatineh AN, Niewiadomska-Bugaj M, Mihalko D (2006) On similarity indices and correction for chance agreement. J Class 23:301-313

Andrich D (1988) The application of an unfolding model of the PIRT type to the measurement of attitude. Appl Psychol Meas 12:33-51

Andrich D, Luo G (1993) A hyperbolic cosine latent trait model for unfolding dichotomous single-stimulus responses. Appl Psychol Meas 17:253-276

Barthélemy J-P, Brucker F, Osswald C (2004) Combinatorial optimization and hierarchical classifications. 4OR 2:179-219

Batagelj V, Bren M (1995) Comparing resemblance measures. J Class 12:73-90

Baulieu FB (1989) A classification of presence/absence based dissimilarity coefficients. J Class 6:233-246

Bertrand P, Diday E (1985) A visual representation of the compatibility between an order and a dissimilarity index: the pyramids. Comput Stat Q 2:31-44

Booth KS, Lueker GE (1976) Testing for the consecutive ones property, interval graphs, and graph planarity using PQ-tree algorithms. J Comput Syst Sci 13:335-379

Braun-Blanquet J (1932) Plant sociology: the study of plant communities (authorized English translation of Pflanzensoziologie). McGraw-Hill, New York

Chepoi V, Fichet B (1997) Recognition of Robinsonian dissimilarities. J Class 14:311-325

Coombs CH (1964) A theory of data. Wiley, New York

Critchley F, Fichet B (1994) The partial order by inclusion of the principal classes of dissimilarity on a finite set, and some of their basic properties. In: Van Cutsem B (ed) Classification and dissimilarity analysis. Springer, New York, pp 5-65 
Dice LR (1945) Measures of the amount of ecologic association between species. Ecology 26:297-302

Diday E (1984) Une représentation visuelle des classes empiétantes: les pyramides. INRIA, research report 291

Diday E (1986) Orders and overlapping clusters in pyramids. In: De Leeuw J, Heiser WJ, Meulman JJ, Critchley F (eds) Multidimensional data analysis. DSWO Press, Leiden, pp 201-234

Fichet B (1984) Sur une extension de la notion de hiérarchie et son équivalence avec quelques matrices de Robinson. Actes des Journées de Statistique de la Grande Motte 12-12

Formann AK (1988) Latent class models for non-monotone dichotomous items. Psychometrika 53:45-62

Fulkerson DR, Gross OA (1965) Incidence matrices and interval graphs. Pac J Math 15:835-855

Ghosh SP (1972) File organization: the consecutive retrieval property. Commun ACM 15:802-808

Gower JC (1986) Euclidean distance matrices. In: De Leeuw J, Heiser WJ, Meulman JJ, Critchley F (eds) Multidimensional data analysis. DSWO Press, Leiden, pp 11-22

Gower JC, Legendre P (1986) Metric and Euclidean properties of dissimilarity coefficients. J Class 3:5-48

Greenberg DS, Istrail S (1995) Physical mapping by STS hybridization: algorithmic strategies and the challenge of software evaluation. J Comput Biol 2:219-273

Hoijtink H (1990) A latent trait model for dichotomous choice data. Psychometrika 55:641-656

Hoijtink H (1991) Parella. Measurement of latent traits by proximity items. DSWO Press, Leiden

Hsu W-L (2002) A simple test for the consecutive ones property. J Algorithms 43:1-16

Hubert L (1974) Some applications of graph theory and related nonmetric techniques to problems of approximate seriation: the case of symmetric proximity measures. Br J Math Stat Psychol 27:133-153

Hubert L, Ararbie P, Meulman J (1998) Graph-theoretic representations for proximity matrices through strongly-anti-Robinsonian or circular strongly-anti-Robinsonian matrices. Psychometrika 63:341-358

Jaccard P (1912) The distribution of the flora in the Alpine zone. New Phytol 11:37-50

Karlin S (1968) Total positivity I. Stanford Univeristy Press, Stanford

Kendall DG (1969) Incidence matrices, interval graphs and seriation in archaeology. Pac J Math 28:565-570

Kendall DG (1971) Seriation from abundance matrices. In: Hodson FR, Kendall DG, Tautu P (eds) Mathematics in the archaeological and historical sciences. University Press, Edinburgh, pp 215-252

Lazarsfeld PF, Henry NW (1968) Latent structure analysis. Mifflin, Houghton

Meidanis J, Porto O, Telles GP (1998) On the consecutive ones property. Discrete Appl Math 88:325-354

Mirkin B (1996) Mathematical classification and clustering. Kluwer, Dordrecht

Mirkin B, Rodin S (1984) Graphs and genes. Springer, Berlin

Post WJ (1992) Nonparametric unfolding models, a latent structure approach. DSWO Press, Leiden

Post WJ, Snijders TAB (1993) Nonparametric unfolding models for dichotomous data. Sonderdruck Methodika 7:130-156

Rasch G (1960) Probabilistic models for some intelligence and attainment tests. Studies in mathematical psychology I.. Danish Institute for Educational Research, Copenhagen

Robinson WS (1951) A method for chronologically ordering archaeological deposits. Am Antiquity 16:293-301

Russel PF, Rao TR (1940) On habitat and association of species of anopheline larvae in South-Eastern Madras. J Malaria Instit India 3:153-178

Schriever BF (1986) Multiple correspondence analysis and ordered latent structure models. Kwantitatieve Methoden 21:117-131

Sibson R (1972) Order invariant methods for data analysis. J R S Soc Ser B 34:311-349

Sijtsma K, Molenaar IW (2002) Introduction to nonparametric item response theory. Sage, Thousand Oaks

Sokal RR, Michener CD (1958) A statistical method for evaluating systematic relationships. Univ Kansas Science Bull 38:1409-1438

Van der Linden WJ, Hambleton RK (eds) (1997) Handbook of modern item response theory. Springer, New York

Warrens MJ (2008a) On similarity coefficients for $2 \times 2$ tables and correction for chance. Psychometrika 73:487-502

Warrens MJ (2008b) On association coefficients for $2 \times 2$ tables and properties that do not depend on the marginal distributions. Psychometrika 73:777-789

Warrens MJ (2008c) On the indeterminacy of resemblance measures for binary (presence/absence) data. J Class 25:125-136

Warrens MJ (2008d) Bounds of resemblance measures for binary (presence/absence) variables. J Class 25:195-208

Warrens MJ, Heiser WJ (2007) Robinson Cubes. In: Brito P, Bertrand P, Cucumel G, Caravalho F de (eds) Selected contributions in data analysis and classification. Springer, Heidelberg, pp 515-523 